\title{
Numerical simulation of magnetic discrete breathers in a Heisenberg spin chain with antisymmetric exchange
}

\author{
I. G. Bostrem ${ }^{1}$, V.E. Sinitsyn ${ }^{1}$, A.S. Ovchinnikov ${ }^{1,2}$, M. I. Fakhretdinov ${ }^{3}$, E. G. Ekomasov ${ }^{\dagger, 3,4}$ \\ †ekomasoveg@gmail.com \\ ${ }^{1}$ Institute of Natural Sciences and Mathematics, Ural Federal University n. a. the first President of Russia B. N. Yeltsin, \\ Yekaterinburg, 620083, Russia \\ ${ }^{2}$ Institute of Metal Physics, Ural Division of the RAS, Yekaterinburg, 620219 Russia \\ ${ }^{3}$ Bashkir State University, Ufa, 450076, Russia \\ ${ }^{4}$ South Ural State University (National Research University), Chelyabinsk, 454080, Russia
}

By using numerical methods, we consider possibility of the spatially localized breather-type excitations for the model of the discrete Heisenberg spin chain, which includes the antisymmetric exchange interaction, the single-ion anisotropy of the easy plane type, and the Zeeman interaction with an external magnetic field, which exceeds a critical field of the transition to the state of forced ferromagnetism. To find solutions, we used equations of motion for spin operators. The case is considered when the frequency of discrete magnetic breathers lies above the upper edge of the spin wave spectrum. The chain length used in the calculations had been taken as 100 and 101 nodes, and the open boundary conditions were used. To carry out numerical calculations, an original program was written which simplifies maximally calculation of spin deviations inside the chain and enables us to use parallel computing technologies. A classification of symmetric and antisymmetric solutions was established, which made it possible to halve a number of calculations for spin deviations. An algorithm was elaborated to specify the amplitudes of spin deviations, that makes possible to construct a desired breather solution in a reasonable amount of time. The numerical calculations of the spin spatial distribution show that it has an antisymmetric ordering with respect to the center of the chain in the presence of the Dzyaloshinskii-Moriya interaction. The center of the solution can be located either between the lattice nodes (Page mode) in the case of an even number of lattice sites, or directly at the node in the case of the odd number (Takeno-Sievers mode). In the first case, the breather mode contains an odd number of pairs of magnetic kinkantikinks with a maximum of the envelope function at the center. Breather modes include an even number of these pairs for an odd number of lattice nodes.

Keywords: Heisenberg spin chain, Dzyaloshinsky-Moriya interaction, magnetic discrete breather, chiral helimagnet.

УДК: 530.182 .1

\section{Численное моделирование дискретных магнитных бризеров в гейзенберговской спиновой цепочке с антисимметричным обменом}

\author{
Бострем И.Г. ${ }^{1}$, Синицын В.Е. ${ }^{1}$, Овчинников А.С. ${ }^{1,2}$, \\ Фахретдинов М.И. ${ }^{3}$, Екомасов Е. Г. ${ }^{\dagger, 3,4}$ \\ ${ }^{1}$ Институт естественных наук и математики, Уральский федеральный университет, Екатеринбург, 620083, Россия \\ ${ }^{2}$ Институт физики металлов, УрО РАН, Екатеринбург, 620219, Россия \\ ${ }^{3}$ Башкирский государственный университет, Уфа, 450076, Россия \\ ${ }^{4}$ Южно-Уральский государственный университет (национальный исследовательский университет), \\ Челябинск, 454080, Россия
}

В работе с помощью численных методов рассмотрена возможность пространственно локализованных возбуждений бризерного типа для модели гейзенберговской спиновой цепочки, которая включает антисимметричное обменное взаимодействие, одноионную анизотропию типа легкая плоскость и зеемановское взаимодействие 
с внешним магнитным полем. Предполагалось, что внешнее магнитное поле превосходит критическое поле перехода в состояние вынужденного ферромагнетизма. Для поиска решений использовалось уравнение движения для спинового оператора. Рассмотрен случай, когда частота возбуждения магнитных дискретных бризеров должна находиться выше верхнего края спектра спиновых волн. Длина цепочки при расчетах имела 100 и 101 узел, использовались открытые граничные условия. При проведении численных расчётов использовался оригинальная программа, максимально упрощающая расчёт спиновых отклонений по цепочке и допускающая возможность использования технологий параллельных вычислений. Целенаправленно строились симметричные и антисимметричные решения задачи, что позволило вдвое сократить число вычислений для спиновых отклонений. Был разработан алгоритм подбора амплитудного спинового отклонения, для которого возможно построение искомого бризерного решения, за разумное время. Численный расчет пространственного распределения спиновых переменных показал, что оно антисимметрично относительно центра цепочки при наличии взаимодействия Дзялошинского-Мория. Центр решения может располагаться либо между узлами решетки (режим Пейджа) в случае их чётного числа, либо непосредственно на узле в случае их нечётного числа (режим Такено-Сиверса). В первом случае бризерная мода содержит нечётное количество пар магнитных кинк-антикинков с максимумом огибающей функции в центре. Бризерные моды включают чётное количество этих пар для нечётного числа узлов решётки.

Ключевые слова: гейзенберговская спиновая цепочка, взаимодействие Дзялошинского-Мория, дискретный магнитный бризер, хиральный гелимагнетик.

\section{1. Введение}

Изучение нелинейной динамики волновых процессов в твердых телах является одним из важнейших направлений в современной физике конденсированного состояния. Нелинейная динамика локализованных волн тесно связана с понятием солитонов как особого типа элементарных возбуждений, определяющим фактором возникновения которых в нелинейных решёточных моделях является дискретность. Было установлено, что в таких системах существуют пространственно локализованные, периодические по времени колебательные режимы, которые были названы дискретными бризерами (ДБ) или внутренними локализованными нелинейными модами $[1,2]$.

До недавнего времени основное внимание при изучении ДБ было сфокусировано на кристаллических решётках [2], хотя имеются экспериментальные свидетельства их появления в других физических системах: оптических волноводах [3], конденсатах Бозе-Эйнштейна [4], гранулированных кристаллах [5] джозефсоновских переходах [6]. В работе [7] сообщалось о наблюдении локализованных нелинейных мод в магнитной среде. Теоретический поиск ДБ в спиновых системах был проведен в работах [8-14], где была продемонстрирована возможность их возникновения в гейзенберговских цепочках с одноионной магнитной анизотропией. Отметим, что имеется определённый пробел в этой области, связанный с учётом более сложных спиновых взаимодействий, в частности, антисимметричного обмена [15], стремящегося стабилизировать гелимагнитное упорядочение магнитных моментов. В данной работе с применением численных методов показано существование нелинейных локализованных возбуждений в модели спиновой цепочки с взаимодействием Дзялошинского-Мория (ДМ) и отмечается связь этого взаимодействия с пространственной симметрией возбуждаемых бризерных мод на цепочках конечной длины.

\section{2. Модель и метод решения}

В качестве модели, являющейся прототипом моноаксиального хирального гелимагнетика [16], рассматриваем цепочку спинов конечной длины $L$, гамильтониан которой выглядит следующим образом:

$$
H=-2 J \sum_{n} \vec{S}_{n} \vec{S}_{n+1}+A \sum_{n}\left(S_{n}^{z}\right)^{2}-H_{0} \sum_{n} S_{n}^{z}+D \sum_{n}\left[\vec{S}_{n} \times \vec{S}_{n+1}\right]_{z},
$$

где $\vec{S}_{n}-$ спиновый вектор $n$-го узла. Здесь первое слагаемое соответствует обменному взаимодействию спинов вдоль оси $z$ с константой взаимодействия $J>0$. Второе - одноионной анизотропии величины $A>0$ при условии, что плоскостью лёгкого намагничивания является плоскость $x y$. Третий член описывает зеемановское взаимодействие спина с внешним магнитным полем $H_{0}$, направленным вдоль оси $z$. Последнее слагаемое отвечает антисимметричному обмену Дзялошинского-Мория с вектором взаимодействия $\mathrm{D}$, направленным вдоль геликоидальной оси. Предполагается, что магнитное поле $H_{0}$ превосходит критическое поле $H_{c r}=2 S\left(\sqrt{4 J^{2}+D^{2}}-J+A\right)$ перехода в состояние вынужденного ферромагнетизма [16]. Здесь $S$ - длина спинового вектора.

Для поиска бризерных решений воспользуемся уравнением движения для спинового оператора $S_{n}^{+}\left(S_{n}^{ \pm}=S_{n}^{x} \pm i S_{n}^{y}\right)$, что даёт соотношение [17]:

$$
\begin{aligned}
& \frac{i \hbar}{2 J S} \frac{d}{d t} s_{n}^{+}=\frac{H_{0}}{2 J S} s_{n}^{+}-2 B s_{n}^{+} s_{n}^{z}+s_{n}^{+}\left(s_{n+1}^{z}+s_{n-1}^{z}\right) \\
& -s_{n}^{z}\left(s_{n-1}^{+}+s_{n+1}^{+}\right)+i \frac{D}{2 J} s_{n}^{z}\left(s_{n-1}^{+}-s_{n+1}^{+}\right)
\end{aligned}
$$

где введены нормированные классические переменные $s_{n}^{ \pm}=S_{n}^{ \pm} / S, s_{n}^{z}=\sqrt{1-s_{n}^{+} s_{n}^{-}}$и константа $B=A / 2 J$.

Используя подстановки $s_{n}^{+}=s_{n}(t) \exp (-i \omega t+i k n a)$, $s_{n}^{z}=\sqrt{1-s_{n}^{2}}$ и выделяя $s$ вещественную и мнимую части, получаем систему уравнений: 


$$
\begin{aligned}
& \Omega s_{n}=-2 B s_{n} \sqrt{1-s_{n}^{2}}+s_{n}\left(\sqrt{1-s_{n+1}^{2}}+\sqrt{1-s_{n-1}^{2}}\right) \\
& -\sqrt{1-s_{n}^{2}}\left(s_{n-1}+s_{n+1}\right) \sqrt{1+\frac{D}{4 J^{2}}} \cos (k a+\delta) \\
& \frac{d s_{n}}{d \tau}=\sqrt{1-s_{n}^{2}}\left(s_{n-1}-s_{n+1}\right) \sqrt{1+\frac{D^{2}}{4 J^{2}}} \sin (k a+\delta),
\end{aligned}
$$

в которой $a-$ постоянная решётки, $k-$ волновой вектор, значение которого находится ниже, $\Omega=\left(\hbar \omega-H_{0}\right) / 2 J S-$ эффективная частота. Очевидно, что в фазе вынужденного ферромагнетизма частота магнитного дискретного бризера должна удовлетворять условию $\hbar \omega>\hbar \omega_{c r}=2 J S \Omega+H_{c r}$. Требование, что частота возбуждения магнитных дискретных бризеров должна находиться выше верхнего края спектра спиновых волн, сводится к условию $\hbar \omega>8 J S \sqrt{1+(D / 2 J)^{2}}$ [16]. Фаза $\delta$ определяется из условия $\operatorname{tg} \delta=D /(2 J)$, а также введено безразмерное время $\tau=t / t_{0}$, где $t_{0}=\hbar /(2 J S)$.

Рассмотрим статические решения в виде магнитного дискретного бризера, когда $d s_{n} / d \tau=0$. Из второго уравнения в этом случае непосредственно следует соотношение $k a=-\operatorname{arctg}(D / 2 J)$, означающее, что волновой вектор дискретного бризера совпадает с соответствующей величиной для конической спирали, которая является основным состоянием гелимагнетика при полях, меньших критического. С целью проследить качественные и количественные различия решений в виде дискретных бризеров, которые могут возбуждаться в хиральной и гейзенберговской спиновой цепочках, численное моделирование для хиральной цепочки будем делать для объекта, который рассматривался ранее без учета антисимметричного обмена [9]. Воспользуемся теми же значениями параметров [9]: длина цепочки - 100 и 101 узел, приведённая константа анизотропии $B=4$, приведённая частота $\Omega=-3.95$, и так же используем открытые граничные условия. Ранее [9] было установлено, что независимо от чётности числа узлов в цепочке возможны нелинейные спиновые возбуждения двух типов - симметричные и антисимметричные относительно отражения от середины цепочки. Также было установлено, что при фиксированных значениях параметров можно построить «иерархию» многосолитонных решений, задавая различные допустимые значения спинового отклонения на краю цепочки.

При проведении численных расчётов использовался алгоритм, отличающийся от использованного в работе [9]. Ввиду того, что при подборе начального спинового отклонения проверку корректности критерия правильности проходится повторять миллионы раз, максимально упростим расчёт спиновых отклонений по цепочке. Вместо численного решения уравнения (2) можно получить из него явное выражение для расчёта $s_{n+1}$ через $s_{n-1}$ и $s_{n}$ :

$$
s_{n+1}=\frac{-A_{n} \sqrt{\left(1-s_{n}^{2}\right)\left(1+\frac{D^{2}}{4 J^{2}}\right)}+\sqrt{1+\left(1-s_{n}^{2}\right) \frac{D^{2}}{4 J^{2}}-A_{n}^{2}}}{1+\left(1-s_{n}^{2}\right) \frac{D^{2}}{4 J^{2}}}
$$

где

$$
A_{n}=s_{n}\left(\Omega-\sqrt{1-s_{n-1}^{2}+2 B \sqrt{1-s_{n}^{2}}}\right)+s_{n-1} \sqrt{1-s_{n}^{2}} \sqrt{1+\frac{D^{2}}{4 J^{2}}} .
$$

В принципе, эта схема позволяет искать как симметричные, так и антисимметричные решения задачи, что позволяет сократить вдвое число вычислений $s_{n}$ за счёт нахождения спиновых отклонений только для одной половины цепочки. Однако, как показывают вычисления, при учете взаимодействия ДзялошинскогоМория остаются только антисимметричные решения, для которых далее описывается способ их построения.

Во-первых, задаём начальное спиновое отклонение для центральных узлов (см. Рис. 1) в цепочке с нечётным числом узлов $(L=2 \cdot N+1)$ полагаем $s_{0}=0, s_{1}=-s_{-1}$; в цепочке с чётным числом узлов $L=2 \cdot N$ задавалось $s_{N+1}=-s_{N}$. Далее, по формуле:

$$
\begin{aligned}
s_{N+2} & =-a \sqrt{1-s_{N+1}^{2}} \sqrt{1+\frac{D}{4 J^{2}}}+ \\
& +s_{N+1} \sqrt{\left(1-s_{N+1}^{2}\right)\left(1+\frac{D^{2}}{4 J^{2}}\right)+s_{N+1}^{2}-a^{2}},
\end{aligned}
$$

где

$$
a=s_{N+1}\left(\Omega+2 B \sqrt{1-s_{N+1}^{2}}-\sqrt{1-s_{N+1}^{2}}-\sqrt{1-s_{N+1}^{2}} \sqrt{1+\frac{D^{2}}{4 J^{2}}}\right)
$$

(цепочка с чётным числом узлов) рассчитывалось спиновое отклонение для соседнего справа узла, затем по общей формуле (3) все остальные значения $s_{n}$ для правой половины цепочки вплоть до последнего $s_{L}$. Для цепочки с нечётным числом узлов можно было сразу пользоваться общей формулой (3), т. к. имеются значения $s_{0}=0$ и задано $s_{1}$. Критерием правильности выбранного значения максимального отклонения, как и в работе [9], служила проверка выполнения уравнения для граничных спинов $s_{L-1}$ и $s_{L}$ :

$$
\Omega s_{L}=s_{L} \sqrt{1-s_{L-1}^{2}}-s_{L-1}^{2} \sqrt{1-s_{L}^{2}}-2 B s_{L} \sqrt{1-s_{L}^{2}} .
$$

Для оценки точности результатов применялась формула для относительной погрешности результата вычисления, которая может быть представлена в следующем виде:

$$
\delta=\frac{\Omega s_{L}-s_{L} \sqrt{1-s_{L-1}^{2}}+s_{L-1}^{2} \sqrt{1-s_{L}^{2}}+2 B s_{L} \sqrt{1-s_{L}^{2}}}{\Omega s_{L}} .
$$

Для получения корректных результатов, как правило, требовалась погрешность менее 0.000001 , что «де факто» соответствует разнице в $0.0001 \%$ между левой и правой частью критерия. Дальнейшее увеличение точности приводило к необходимости рассматривать диапазон с более мелким шагом от стартового значения $S$, что существенно удлиняло расчёт. Как известно, состояния солитонного типа возникают при строго определённом наборе параметров (при фиксированных $B$ и $\Omega$ точное значение должно принимать начальное отклонение). Как показа- 


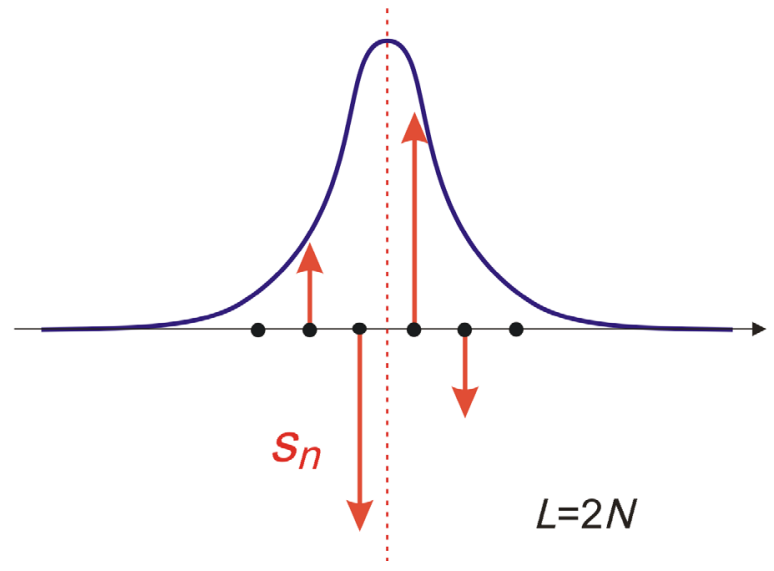

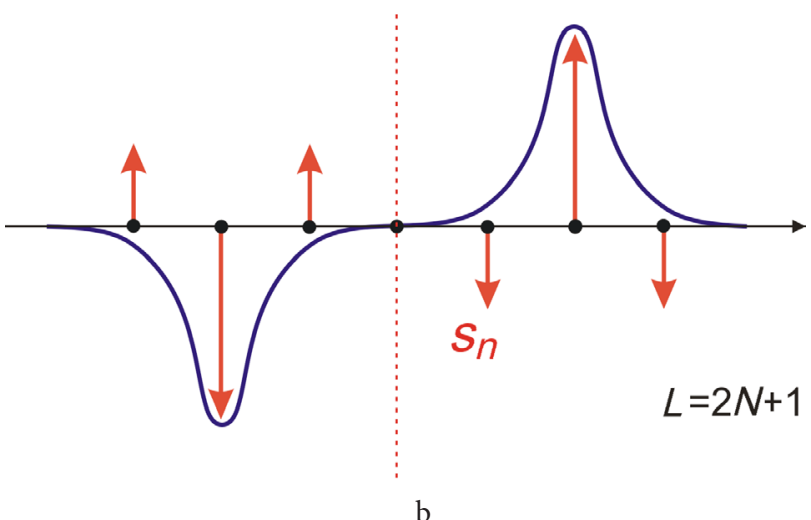

b

Pис. 1. (Color online) Схема антисимметричного расположения спиновых переменных относительно центра цепочки (красная пунктирная линия) при наличии ДМ-взаимодействия: чётное (а) и нечётное (b) количество узлов решётки. Сплошная синяя линия показывает профиль огибающей функции.

Fig. 1. (Color online) Scheme of the antisymmetric arrangement of spin variables with respect to the center of the chain (red dashed line) in the presence of DM interaction: an even (a) and an odd (b) number of lattice sites. The solid blue line shows the profile of the envelope function.

ли результаты численного моделирования, оно должно подбираться с точностью до 6-8 знаков. Для длины цепочки 100-101 узлов приходится перебирать и проверять около $2 \cdot 10^{9}$ значений. Был разработан алгоритм подбора амплитудного спинового отклонения, для которого возможно построение искомого бризерного решения, за разумное время. Поскольку используемая программа производит расчёт всей цепочки и проверку выполнения граничного уравнения на её конце, она допускает возможность использования технологий параллельных вычислений, поскольку все потоки процессов могут выполняться независимо и не имеют пересекающихся переменных. Единственное отличие одного потока выполнения от другого - стартовое число $S_{0}$. Математические особенности задачи таковы, что подбор значений параметров начального отклонения необходимо производить с большой точностью (в плане количества знака после запятой) и при этом искать решения в широком диапазоне. Для этого в программе были выбраны следующие условия: за один запуск программа проверяла $2 \cdot 10^{9}$ точек от $S_{0}$ заданного до $S_{0}+S_{0} / 2 \cdot 10^{9}$, а каждый поток вычислений обрабатывал стартовое значение $S=S_{0}+S_{0} \cdot\left(\right.$ номер потока) $/ 2 \cdot 10^{9}$, проверяя для неё корректность выполнения граничного уравнения с заданной точностью. Если уравнение выполнялось, программа записывала значение $S$ и выводила его на экран. Для ускорения процесса была применена технология параллельных вычислений на центральном процессоре OpenMP. При необходимости, программа может быть оптимизирована для расчётов на видеокарте, но при решении рассматриваемой задачи такая оптимизация не потребовалась.

Численных расчет пространственного распределения спиновых переменных $s_{n}$ показал, что оно антисимметрично относительно центра цепочки при наличии взаимодействия Дзялошинского-Мория (симметричные решения также получено, но для случая нулевого ДМ).
Центр решения может располагаться либо между узлами решетки (режим Пейджа [18]) в случае их чётного числа, $L=2 N$, либо непосредственно на узле в случае $L=2 N+1$ (режим Такено-Сиверса [19]). В первом случае (см. Рис. 1a) бризерная мода содержит нечётное количество пар магнитных кинк-антикинков с максимумом огибающей функции в центре. Бризерные моды включают чётное количество этих пар для нечётного числа узлов решётки (Рис. 1b). Пространственное распределение спиновых моментов, полученных с помощью численного решения уравнений (3), показано на Рис. 2 как без учёта взаимодействия Дзялошинского-Мория, так и при его наличии для различного числа пар кинк-антикинк. В случае нулевого параметра $D$ поперечные компоненты спинов упорядочены строго антиферромагнитным образом внутри области локализации каждой пары кинк-антикинк. При ненулевых значениях $D$ возникает вращательная модуляция этого антиферромагнитного упорядочения с волновым вектором $k a=-\operatorname{arctg}(D / 2 J)$. Как видно из этого рисунка антисимметричный обмен нарушает инверсионную симметрию расположения связанных пар магнитных кинк-антикинк относительно центра системы. В работе [9] отмечалось, что можно построить «иерархию» бризерных решений, бризерные решетки с 1,2..6 солитонами, но авторы не изучали, сколько таких решений можно получить для цепочки с заданной длиной. Диапазон изменения амплитуды центрального спинового отклонения можно оценить следующим образом: нижнее значение позволяет получить аналитическое решение в континуальном приближении [17]. Минимальная амплитуда спинового отклонения отвечает односолитонному решению с максимальным периодом, который обычно превышает длину цепочки. С увеличением числа солитонов в решетке амплитуда растет, а период уменьшается. Верхнее значение можно оценить с позиций здравого смысла: период не может быть меньше постоянной решетки. 


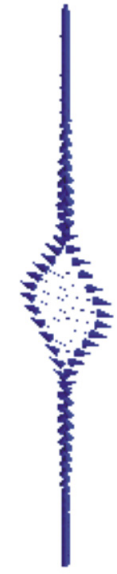

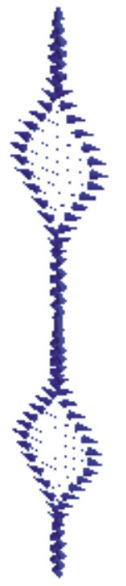

$\mathrm{b}$

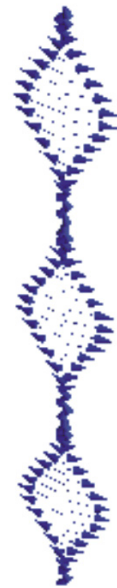

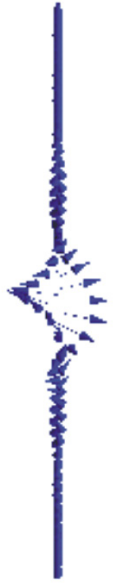

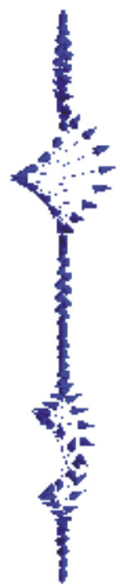

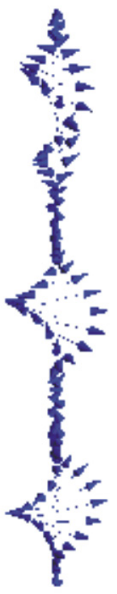

f

Рис. 2. Пространственное распределение спиновых моментов (синие стрелки) в спиновой цепочке с различным числом пар кинк-антикинк. Случаи без взаимодействия Дзялошинского-Мория: $n=1$ с областью локализации 60 узлов (а), $n=2$ с областью локализации для каждой пары 50 узлов (b), $n=3-35$ узлов (c). Случаи с учетом ДМ-взаимойдействия и такими же областями локализации: $n=1$ (d), $n=2$ (e), $n=3$ (f). Параметры $B=4.0, \Omega=-3.95$. Длина цепочки 100 или 101 узел.

Fig. 2. Spatial distribution of spin moments (blue arrows) in a spin chain with different numbers of kink-antikink pairs. Cases without Dzyaloshinskii-Moryia interaction: $n=1$ with localization scale of 60 sites (a), $n=2$ with localization area 50 sites for the each pair (b), $n=3-35$ sites (c); and with an account of the DM interaction and the same localization areas: $n=1$ (d), $n=2$ (e), $n=3$ (f). The parameters $B=4.0, \Omega=-3.95$ are taken. The length of chain is either 100 or 101 sites.

Табл. 1. Максимальное спиновое отклонение для кноидальных решеток с различным числом пар кинк-антикинк.

Table 1. Maximum spin deviation for cnoidal lattices with different numbers of kink-antikink pairs.

\begin{tabular}{|c|c|c|}
\hline $\begin{array}{c}\text { Число пар кинк-антикинк } \\
\text { (число солитонов) } \\
\begin{array}{c}\text { Number of kink-antikink pairs } \\
\text { (number of solitons) }\end{array}\end{array}$ & $\begin{array}{c}\text { Без учета антисимметричного обмена } \\
\text { Excluding antisymmetric exchange }\end{array}$ & $\begin{array}{c}\text { С учетом антисимметричного обмена } \\
\text { Taking into account the antisymmetric exchange }\end{array}$ \\
\hline 1 & 0.223607 & 0.156404 \\
\hline 2 & 0.223628 & 0.157228 \\
\hline 3 & 0.224478 & 0.161817 \\
\hline 4 & 0.228027 & 0.173050 \\
\hline 5 & 0.234822 & 0.188055 \\
\hline 6 & 0.247389 & 0.201659 \\
\hline 7 & 0.262284 & 0.224179 \\
\hline
\end{tabular}

Так как значение «затравочного» отклонения меняется дискретно, можно ожидать, что число возможных возбуждений-бризеров будет конечным. Результаты численного моделирования это подтверждают, однако окончательный вывод о конечности числа возможных типов возбуждений требуют дальнейших исследований. Кроме этих качественных отличий имеются и количественные: как видно из Табл. 1 наличие антисимметричного обмена приводит к уменьшению амплитуды отклонений. Видно, что изменение уже третьего знака приводит к качественным изменением в решении. Проведенное численное моделирование нелинейных возбуждений рассматриваемого типа в хиральной цепочке показало, при учете антисимметричного обмена возможно возбуждение только антисимметричных дискретных бризеров (см. Рис. 2).

\section{3. Заключение}

Исследованы пространственно локализованные бризерные возбуждения для модели гейзенберговской спиновой цепочки, которая включает антисимметричное обменное взаимодействие и одноионную анизотропию типа легкая плоскость. Показано, что в цепочке конечного размера магнитные бризерные моды могут быть проиндексированы по количеству вложенных пар кинк-антикинков, образующих регулярную бризерную решетку. Учет антисимметричного обмена приводит к возбуждению только антисимметричных магнитных дискретных бризеров.

Благодарности / Akcnowledgements. Работа выполнена при поддержке гранта РФФИ (проект No. 20-02-00213). / 
This work was supported by a grant from the Russian Foundation for Basic Research (project No. 20-02-00213).

\section{Литература/References}

1. S. Flach, C. R. Willis. Phys. Rep. 295, 181 (1998). Crossref

2. S.V. Dmitriev, E.A. Korznikova, J.A. Baimova, M. G. Velarde. Phys. Usp. 59, 446 (2016). Crossref

3. R. Morandotti, U. Peschel, J. Aitchison, H. Eisenberg, Y. Silberberg. Phys. Rev. Lett. 83, 2726 (1999). Crossref

4. B. Eiermann, T. Anker, M. Albiez, M. Taglieber, P. Treutlein, K.-P. Marzlin, M.K. Oberthaler. Phys. Rev. Lett. 92, 230401 (2004). Crossref

5. N. Boechler, G. Theocharis, S. Job, P. Kevrekidis, M. Porter, C. Daraio. Phys. Rev. Lett. 104, 244302 (2010). Crossref

6. E. Trias, J. Mazo, T.P. Orlando. Phys. Rev. Lett. 84, 741 (2000). Crossref

7. D. Backes, F. Macia, S. Bonetti, R. Kukreja, H. Ohldag, A. D. Kent. Phys. Rev. Lett. 115, 127205 (2015). Crossref

8. R. F. Wallis, D. L. Mills, A. D. Boardman. Phys. Rev. B. 52, R3828 (1995). Crossref

9. S. Rakhmanova, D. L. Mills. Phys. Rev. B. 54, 9225 (1996). Crossref
10. R. Lai, S. A. Kiselev, A. J. Sievers. Phys. Rev. B. 54, R12665 (1996). Crossref

11. W. Su, J. Xie, T. Wu, B. Tang. Chin. Phys. B. 27, 097501 (2018). Crossref

12. Z. I. Djoufack, J.P. Nguenang, A. Kenfack-Jiotsa. Physica B. 598, 412437 (2020). $\underline{\text { Crossref }}$

13. M. Lakshmanana, Avadh Saxena. Phys. Lett. A. 382, 1890 (2018). Crossref

14. R. S. Kamburova, S. K. Varbev, M. T. Primatarowa. Phys. Lett. A. 383, 471 (2019). Crossref

15. L. Kavitha, E. Parasuraman, D. Gopi, A. Prabhu, R. A. Vicencio. J. Magn. Magn. Mater. 401, 394 (2016). Crossref

16. J. Kishine J., A.S. Ovchinnikov. Theory of Monoaxial Chiral Helimagnet. In: Solid State Physics, vol. 66 (ed. by R. E. Camley and R. L. Stamps). Academic Press, New York (2015), pp. 1-130. Crossref

17. I.G. Bostrem, E.G. Ekomasov, J.-i. Kishine, A.S. Ovchinnikov, V.E. Sinitsyn. Chelyab. Fiz.-Mat. Zh. 5 (2), 194 (2020). Crossref

18. J. B. Page. Phys. Rev. B. 41, 7835 (1990). Crossref

19. A. Sievers, S. Takeno. Phys. Rev. Lett. 61, 970 (1988). Crossref 